\section{shRNA: solving the subtle}

\section{By Lev Osherovich, Senior Writer}

A report published in the Proceedings of the National Academy of Sciences illustrates how subtle structural differences between two types of RNAs - artificial small hairpin RNA and endogenous microRNA-may help explain the difference between shRNA having a therapeutic effect or being toxic. ${ }^{1}$

The researchers found that designing miRNA-like shRNAs offered a potential solution to shRNA toxicity. But industry researchers approached by SciBX also emphasized the need for a solid initial shRNA design process to avoid unwanted side effects in the first place.

shRNAs consist of a double-stranded stretch of RNA (21 nucleotides in length) capped off on one end by a single-stranded RNA loop (6-8 nucleotides long). The active moiety, the 21-nucleotide guide strand from the double-stranded portion of the shRNA, is complementary to a target mRNA. The remaining strand is called the passenger strand.

shRNA molecules can be expressed from transgenic or viral vectors and can also be introduced into cells exogenously. Once inside the cytoplasm, an shRNA is split by the cellular machinery into the singlestranded guide and passenger strands. The guide strand proceeds to form an RNA-protein complex called the RNA-induced silencing complex (RISC), which cleaves its complementary mRNA. The result is reduced expression of the corresponding protein (see Figure 1, "Staking out the RNAi landscape," page 6).

In the PNAS study, a team led by Beverly Davidson, professor of neurology at the University of Iowa, assessed the feasibility of using shRNA to treat Huntington's disease in mice expressing the mutant form of huntingtin that is responsible for the disease. The researchers tested various shRNA constructs delivered by adenoassociated viral (AAV) vectors and determined changes in levels of the toxic protein.

The researchers began by testing a panel of 35 shRNA constructs that target different parts of the huntingtin gene, looking for reductions in huntingtin mRNA levels in mouse and human cell cultures. The three best candidates were injected into the brains of transgenic mice with HD. Although all three reduced huntingtin mRNA levels, two of them also triggered astroglial inflammation and striatal toxicity.

"We set out to test the hypothesis of whether or not we could reduce huntingtin expression in the adult brain," Davidson told SciBX. But "the finding that some of these shRNAs are toxic came as a surprise."

\section{Controlling the guide}

Suspecting that the structure of the artificial shRNA may be at the root of the toxicity, Davidson's team developed an alternative, more natural miRNA-based approach to silencing the huntingtin gene. Only recently discovered, miRNAs are important endogenous regulators of gene expression and are the natural substrates for the enzymes that process shRNAs and other RNAi constructs into small interfering RNAs, the double-stranded RNAs consisting of the guide and the passenger strands.

The team developed miRNA vectors expressing guide strands equivalent to those encoded by the toxic shRNAs. The structure of the artificial miRNAs expressed by these vectors resembles that of naturally occurring miRNAs, which is characterized by sequence tails protruding beyond their guide strands; by contrast, shRNAs do not have such overhangs.

Davidson hypothesized that the toxicity observed with her group's shRNAs may have been caused by high levels of specific guide strands, which can result in nonspecific mRNA targeting ${ }^{2}$ or problems with the processing of natural miRNAs. ${ }^{3}$

In experiments with the miRNA vectors and using the same mouse model as before, the team saw marked reductions in guide strand accumulation and toxicity compared with the original shRNA experiments while still achieving similar drops in the levels of huntingtin mRNA.

University of lowa

Why the high levels of shRNA guide strands had accumulated in the first place remained unanswered, though.

Johannes Fruehauf, VP of research at shRNA company Cequent Pharmaceuticals Inc., told SciBX that if the toxicity in Davidson's study is a result of the high levels of guide strand made by the original shRNA constructs, companies should be wary of boosting the levels of their shRNA therapeutics.

"People are trying to maximize activity, but you may need to stay away from the threshold" of toxicity, he said.

Fruehauf added that the Davidson study is another piece of evidence pointing to potentially dangerous off-target effects of RNAi therapeutics. "People will look into off-target effects much more seriously as a result of this paper," he told SciBX. "Off-target effects are an important threat to the field, and we all must study ways to recognize and minimize them."

But Fruehauf also noted the toxicity could have arisen during the actual processing of the shRNA transcripts from the AAV vectors rather than from a more general problem with shRNAs per se.

Davidson agreed that the higher levels of guide strand derived from toxic shRNA compared with safe shRNAs could account for her results. However, the toxic shRNAs also killed neurons at low vector titers, suggesting that an additional dose-independent mechanism of toxicity may be involved.

Peter Lindsey, CSO at miRNA therapeutic developer Regulus Therapeutics LLC, had a different take on the data. He pointed to the 
passenger strand, the mirror image of the guide strand that is ordinarily discarded during siRNA processing, as a likely suspect in toxicity.

Lindsey noted that in the in vitro portion of the Davidson study, the most toxic shRNAs left behind higher levels of passenger-strand RNAs than did the nontoxic shRNAs. Although the guide strand targets huntingtin mRNA, passenger strands could be targeting unrelated mRNAs for degradation, leading to off-target effects.

"The take home message is that if you don't have good shRNA, and don't design your sequences correctly, you can have a lot of passenger strand activity. That can sometimes have deleterious effects," said Lindsey.

He suggested that although the study shows using miRNA vectors can reduce toxicity, a better design of the original shRNA constructs could avoid the problem. According to Lindsey, the solution is to screen genomic sequence databases for possible off-pathway targets of passenger strands prior to designing shRNA therapeutics.

However, according to Davidson, the passenger strands of the toxic shRNAs were virtually undetectable in vivo, and thus any off-target effects of passenger strands would have required "very potent" siRNA activity by a very small number of molecules.

Davidson and Barrie Carter, EVP and CSO of Targeted Genetics Corp. and an author on the PNAS paper, told SciBX that the most effective, nontoxic miRNA constructs identified in the study are now being tested for long-term safety and efficacy in the mouse model of HD. The effect of huntingtin mRNA depletion on neuropathology and disease progression in miRNA-treated mice will be reported in a forthcoming publication, Davidson said.

\section{Staying the RNAi course}

Regardless of the mechanism of shRNA toxicity, other companies in the space told SciBX that they don't foresee any effects of Davidson's observations on their R\&D programs.

John Maraganore, CEO of Alnylam Pharmaceuticals Inc., noted that shRNAs "have a distinct stem loop structure" and that the miRNA workaround used by the researchers to avoid toxicity may be relevant to "the specific design and construction of shRNAs" rather than to RNAi technology in general.

Alnylam, in partnership with Medtronic Inc., is developing ALNHTT, a therapeutic siRNA in preclinical development to treat HD. In a 2007 PNAS article, researchers at Massachusetts General Hospital reported that ALN-HTT was safe and reduced pathology in a mouse model of HD. ${ }^{4}$

Alnylam's lead compound is ALN-RSV01, an intranasal siRNA therapeutic in Phase II testing to treat respiratory syncytial virus (RSV) infection.

In contrast, Cequent's technology generates shRNA molecules using bacterial vectors, which enter target cells and deliver their cargo to the cytoplasm, according to Fruehauf. The resulting shRNA is subsequently processed by the cell enzymes into siRNA. This approach "avoids the problems with making the target cells the source of the shRNA" through transfection or gene therapy.

The company's lead compound, Cequent 501, is in preclinical testing to treat familial adenomatous polyposis (FAP).

Finally, the AAV vector-driven miRNA approach developed by Davidson and Targeted Genetics may represent an RNAi platform that provides an integrated solution to three of the main challenges in the field. According to Carter, an AAV vector that produces miRNAs "solves the delivery issues, the inflammation issues and the off-target issues" that could hinder other approaches.

Carter thinks that Targeted Genetics' strategy of expressing siRNAs from transfected AAV vectors allows targeting of cell populations in hard-to-reach places such as the brain without triggering an innate immune response, a problem commonly associated with adding synthetic siRNAs directly to cells (see What's new, or not, in siRNA, page 5).

The PNAS study was performed in collaboration with Targeted Genetics and Sirna Therapeutics Inc., a wholly owned subsidiary of Merck \& Co. Inc. Earlier this month, Targeted Genetics acquired Merck's share of the companies' jointly developed preclinical HD program in exchange for undisclosed royalties. The acquisition also included exclusive, worldwide rights to IP related to AAV vector-expressed RNAi that Sirna had licensed from the University of Iowa.

The technology described in Davidson's paper is patented by the university and has been licensed to Targeted Genetics. In addition, the company has filed for patents for the use of AAV vectors in siRNA therapeutics for HD.

REFERENCES

1. McBride, J.L. et al. Proc. Natl. Acad. Sci. USA; published online April 7, 2008; doi:10.1073/pnas.0801775105

Contact: Beverley L. Davidson, University of lowa, lowa City, lowa e-mail: beverly-davidson@uiowa.edu

2. Ma, Y. et al. Nature 443, 359-363 (2006)

3. Grimm, D. et al. Nature 441, 537-541 (2006)

4. Difiglia, M. et al. Proc. Natl. Acad. Sci. USA 104, 17204-17209 (2007)

COMPANIES AND INSTITUTIONS MENTIONED

Alnylam Pharmaceuticals Inc. (NASDQ:ALNY), Cambridge, Mass. Cequent Pharmaceuticals Inc., Cambridge, Mass.

Massachusetts General Hospital, Boston, Mass.

Medtronic Inc. (NYSE:MDT), Minneapolis, Minn.

Merck \& Co. Inc. (NYSE:MRK), Whitehouse Station, N.J.

Regulus Therapeutics LLC, Carlsbad, Calif.

Sirna Therapeutics Inc., San Francisco, Calif.

Targeted Genetics Corp. (NASDAQ:TGEN), Seattle, Wash. University of lowa, lowa City, lowa 